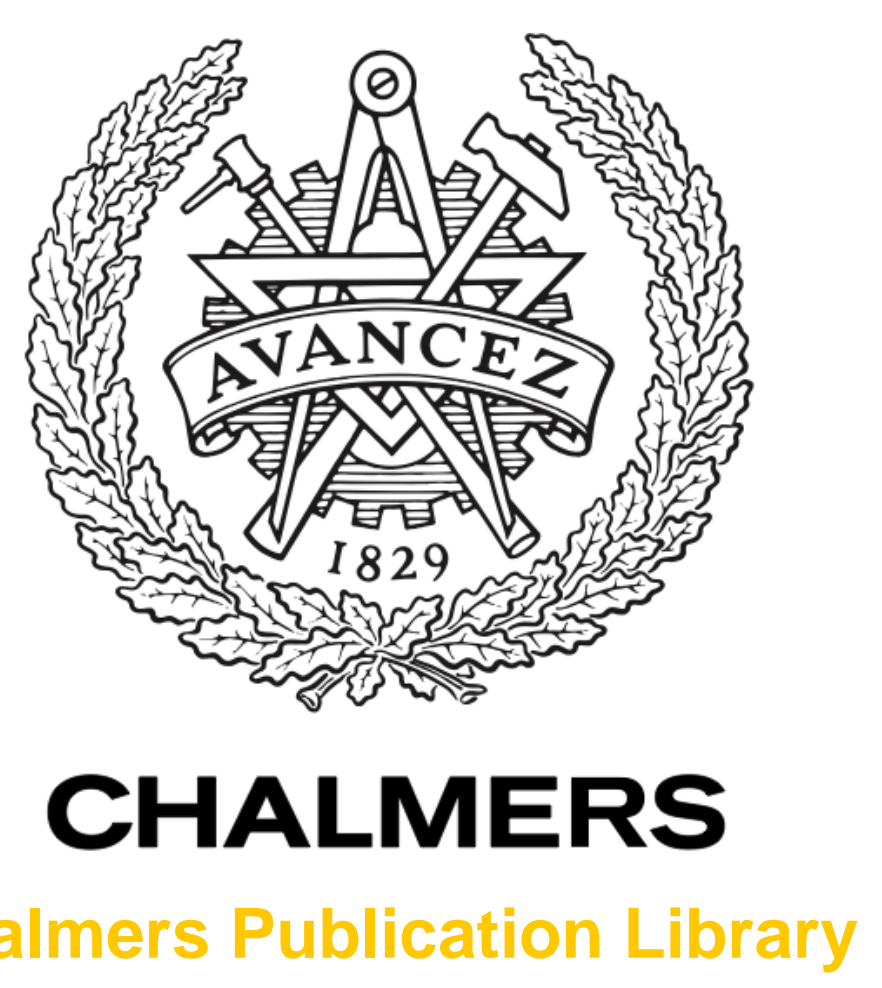

Chalmers Publication Library

\author{
Fission Yields of Minor Actinides at Low Energy Through Multi-nucleon Transfer \\ Reactions of $<$ sub $>238</$ sub $>$ U on $<$ sub $>12</$ sub $>$ C
}

This document has been downloaded from Chalmers Publication Library (CPL). It is the author's version of a work that was accepted for publication in:

Acta Physica Polonica B (ISSN: 0587-4254)

Citation for the published paper:

Ramos, D. ; Rodriguez-Tajes, C. ; Caamano, M. et al. (2015) "Fission Yields of Minor

Actinides at Low Energy Through Multi-nucleon Transfer Reactions of <sub $>238</$ sub $>\mathrm{U}$

on $<$ sub $>12</ s u b>C "$. Acta Physica Polonica B, vol. 46(3), pp. 443-446.

http://dx.doi.org/10.5506/APhysPolB.46.443

Downloaded from: http://publications.lib.chalmers.se/publication/218441

Notice: Changes introduced as a result of publishing processes such as copy-editing and formatting may not be reflected in this document. For a definitive version of this work, please refer to the published source. Please note that access to the published version might require a subscription.

Chalmers Publication Library (CPL) offers the possibility of retrieving research publications produced at Chalmers University of Technology. It covers all types of publications: articles, dissertations, licentiate theses, masters theses, conference papers, reports etc. Since 2006 it is the official tool for Chalmers official publication statistics. To ensure that Chalmers research results are disseminated as widely as possible, an Open Access Policy has been adopted.

The CPL service is administrated and maintained by Chalmers Library. 


\section{FISSION YIELDS OF MINOR ACTINIDES AT LOW ENERGY THROUGH MULTI-NUCLEON TRANSFER REACTIONS OF ${ }^{238} \mathrm{U}^{\mathrm{ON}}{ }^{12} \mathrm{C}^{*}$}

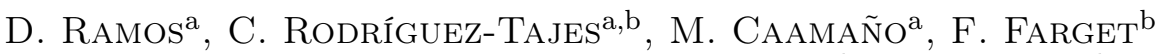
L. Audouin ${ }^{\mathrm{c}}$, J. Benlliure $^{\mathrm{a}}$, E. Casarejos $^{\mathrm{d}}$, E. Element $^{\mathrm{b}}$

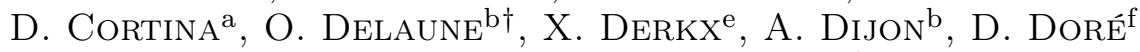
B. Fernández-Domínguez ${ }^{\mathrm{a}}$, G. De France ${ }^{\mathrm{b}}$, A. Heinz $^{\mathrm{g}}$ B. Jacquot ${ }^{b}$, A. Navin ${ }^{b}$, C. Paradela ${ }^{a}$, M. Rejmund $^{b}$, T. Roger $^{b}$ M.D. SALSAC ${ }^{\mathrm{f}}$, C. SCHMitT ${ }^{\mathrm{b}}$

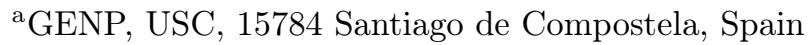

${ }^{\mathrm{b}}$ GANIL, CEA/DMS-CNRS/IN2P3, BP 55027, 14076 Caen Cedex 05, France 'IPN Orsay, Université de Paris-Sud, CNRS/IN2P3, 91406 Orsay Cedex, France ${ }^{\mathrm{d}}$ CIMA, University of Vigo, 36310 Vigo, Spain

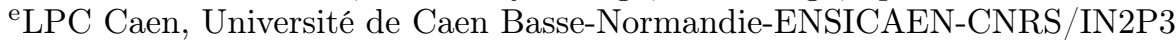
14050 Caen Cedex, France

${ }^{\mathrm{f}} \mathrm{CEA}$ Saclay, DMS/IRFU/SPhN, 91191 Gif-sur-Yvette Cedex, France ${ }^{\mathrm{g}}$ Chalmes University of Technology, 41296 Göteborg, Sweden

(Received January 12, 2015)

First preliminary results of fragments distribution as a function of the excitation energy for different fissioning systems from $\mathrm{U}$ to $\mathrm{Cm}$ and ${ }^{250} \mathrm{Cf}$ are presented. A new method based on inverse kinematics to study transferinduced fission of minor actinides was carried out in GANIL in 2008, and again in 2011. In these experiments, a ${ }^{238} \mathrm{U}$ beam at $6.1 \mathrm{AMeV}$ impinged on a carbon target to produce fissioning systems by multi-nucleon transfer reactions, resulting in the first experiments accessing the full identification of a collection of fissioning systems and their corresponding fission fragments distribution. The excitation energy is deduced from the detection of the recoil nucleus in an angular DE-E stripped silicon telescope, and the identification of the fragments is made possible by the VAMOS spectrometer.

DOI:10.5506/APhysPolB.46.443

PACS numbers: 25.70.Jj, 24.50.+g, 25.85.Ge, 29.85.-c

* Presented at the Zakopane Conference on Nuclear Physics "Extremes of the Nuclear Landscape", Zakopane, Poland, August 31-September 7, 2014.

† Present address: CEA DAM DIF, 91297 Arpajon, France.

‡ Present address: EC-JRC-IRMM, Retieseweg 111, 2440 Geel, Belgium. 


\section{Introduction}

Fission is the largest scale collective motion of the nucleons inside the nucleus while, at the same time, at low excitation energies, it is strongly influenced by the microscopic structure of the nucleus [1-3]. An accurate description of the fission remains challenging due to the complex nature of the process.

The present work provides a new ensemble of measurements that relates the excitation energy of the fissioning system, produced either by fusion or transfer reactions, with the full fragment distribution. These new measurements for fissioning systems not accesible with other techniques also provide nuclear data, important for nuclear energy applications, such as the development of new generation nuclear reactors.

\section{Experimental setup}

A ${ }^{238} \mathrm{U}$ beam with an energy of $6.14 \mathrm{AMeV}$ impinged on a $100 \mu \mathrm{g} / \mathrm{cm}^{2}$ ${ }^{12} \mathrm{C}$ target. Transfer reactions produced heavy actinides with a characteristic excitation-energy distribution. The target-like nucleus was detected and identified by using a telescope, composed by two double-sided, annular, Si detectors. The segmentation of the detector provided its scattering angle, which with the measurement of its energy, allowed the reconstruction of the transfer reaction and the excitation energy [4]. In the absence of target-like recoil detection, a fusion reaction is assumed.

The corresponding actinide may decay by fission. In such case, its products are fully identified in atomic and mass number, and in charge state by a detection set at the focal plane of the spectrometer VAMOS. This detection set comprises a multiwire chamber for the time-of-flight measurements, two drift chambers for position and angle determination, and an ionization chamber and an Si wall to perform energy-loss and residual energy measurements. The angles at the target position and the energy of the fission fragments are also reconstructed by back-tracing methods [5]. The correction of the VAMOS acceptance is applied for each (A,Q)-system individually [6].

\section{Fission products}

Four different fissioning systems are reported in this work: ${ }^{250} \mathrm{Cf}$ with a well-defined excitation energy of $45 \mathrm{MeV},{ }^{240} \mathrm{Pu},{ }^{239} \mathrm{~Np}$ and ${ }^{238} \mathrm{U}$ with a distribution of excitation energy centered in $11 \mathrm{MeV}, 7.5 \mathrm{MeV}$ and $8 \mathrm{MeV}$, respectively, with a full width at half maximum of approximately $8 \mathrm{MeV}$ in all the cases [4]. Figure 1 (left) shows the fission yields as a function of the fission-fragment atomic number for these systems. It can be observed how the contribution of different fission modes varies. ${ }^{250} \mathrm{Cf}$ mainly presents a 
symmetric fission in contrast to the rest of the systems, where asymmetric fission is dominant. ${ }^{238} \mathrm{U}$ is affected, in less than $10 \%$, by contamination of ${ }^{250} \mathrm{Cf}$ due to random coincidences between elastic scattering with fusionfission events. In Fig. 1 (right), the fission yields of ${ }^{240} \mathrm{Pu}$ for three ranges of excitation energy, $7 \pm 2,11 \pm 2$, and $15 \pm 2 \mathrm{MeV}$, are presented. The fact that the valley between the two maxima becomes less deep when increasing the excitation energy reflects the damping of the shell effects that are expected to be more relevant at lower excitation energies. A calculation with the GEF code (a GEneral description of Fission observables) [8] is compared with the data and a good agreement is found.
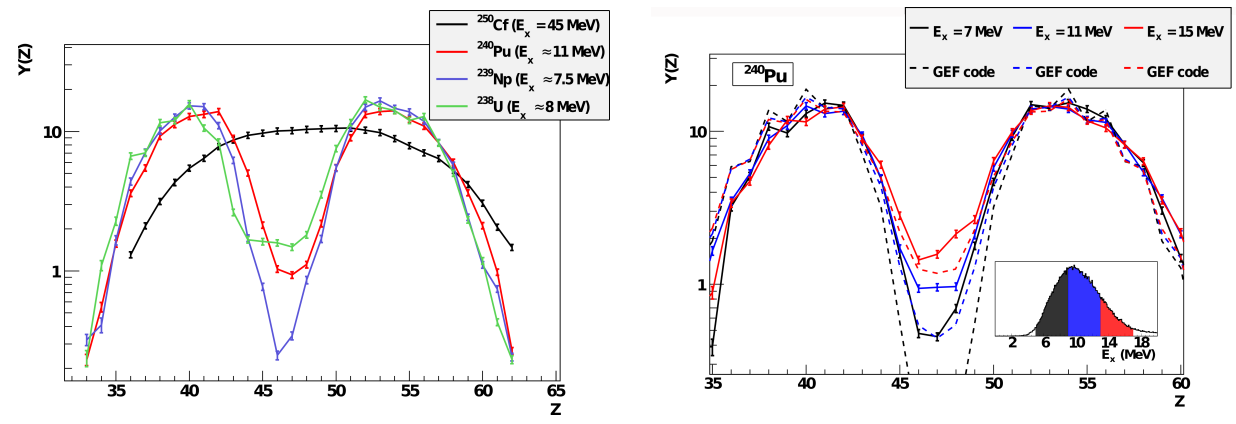

Fig. 1. Atomic number distribution of the fission fragments. (Left) Different fissioning systems are shown. (Right) The case of ${ }^{240} \mathrm{Pu}$ with different ranges of excitation energy, compared with GEF calculations. (Inset) Excitation energy distribution.
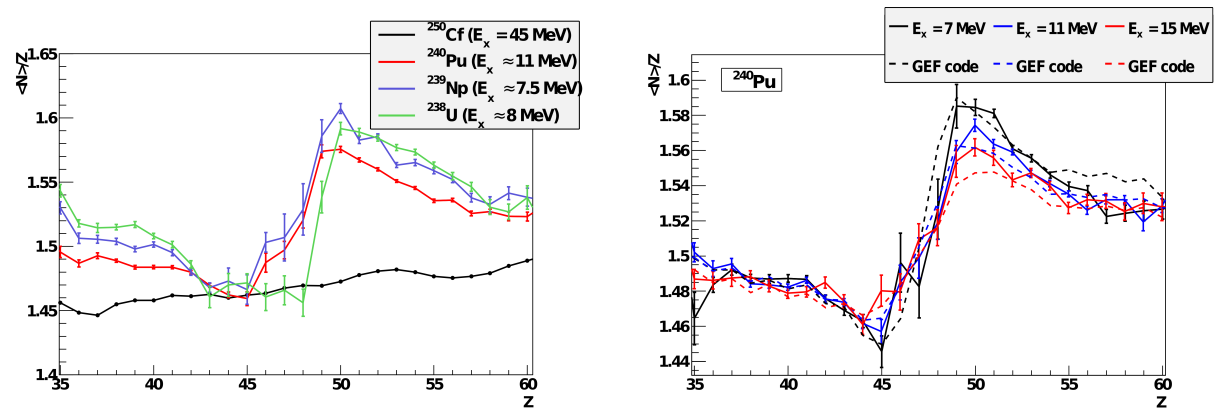

Fig. 2. Neutron excess as a function of the atomic number of the fission fragments. (Left) Different fissioning systems are shown. (Right) The case of ${ }^{240} \mathrm{Pu}$ with different ranges of excitation energy, compared with GEF calculations.

Figure 2 shows the neutron excess of the fission fragments, defined as the ratio of the average neutron number of the fission products post neutron evaporation over its atomic number. On the left-hand side, this is presented 
for the different fissioning systems. The observed sawtooth behavior in the neutron excess reveals shell effects that are independent of the fissioning system. On the right-hand side, three ranges of excitation energy are selected for ${ }^{240} \mathrm{Pu}$. It is shown how this sawtooth behavior becomes smoother by increasing the excitation energy, which is coherent with the damping of shell effects. The variation of the neutron excess with the excitation energy is larger in the region of $\mathrm{Z} \approx 50$. GEF calculations reproduce accurately the behavior of the neutron excess for the case of ${ }^{240} \mathrm{Pu}$.

\section{Summary and outlook}

This experiment allowed the study of four different fissioning systems ${ }^{238} \mathrm{U},{ }^{239} \mathrm{~Np},{ }^{240} \mathrm{Pu}$ and ${ }^{250} \mathrm{Cf}$, by identifying the transfer channels and reconstructing the excitation energy before the fission process, and measuring the fission products with the VAMOS spectrometer.

An evolution of the valley of the fragment distribution for the systems with low excitation energy was observed. This valley is deeper for lower excitation energies. The investigation of the neutron excess of the fragments reflects nuclear-structure effects that decrease by increasing the excitation energy.

Finally, more observables such as fragment velocities, total kinetic energy, and even-odd effect will become available as the analysis progresses, providing a complete set of observables for a detailed description of the fission process.

This work was partially supported by the Spanish Ministry of Research and Innovation under the Grants No. FPA2010-22174-C02-01, RYC-201211585, and BES-2011-045183.

\section{REFERENCES}

[1] J.L. Sida et al., Nucl. Phys. A502, 233c (1989).

[2] S. Steinhäuser et al., Nucl. Phys. A634, 89 (1998).

[3] C. Böckstiegel et al., Nucl. Phys. A802, 12 (2008).

[4] C. Rodríguez-Tajes et al., Phys. Rev. C89, 24614 (2014).

[5] M. Rejmund et al., Nucl. Instrum. Methods Phys. Res. A646, 184 (2011).

[6] M. Caamaño et al., Phys. Rev. C88, 24605 (2013).

[7] S. Pullanhiotan et al., Nucl. Instrum. Methods Phys. Res. A593, 343 (2008).

[8] K.-H. Schmidt, B. Jurado, JEF/DOC 1423, 2012. 